Postprint version. Please refer to the publisher's version as follows:

Zapounidou S., Sfakakis M., Papatheodorou C. (2014) Library Data Integration: Towards BIBFRAME Mapping to EDM. In: Closs S., Studer R., Garoufallou E., Sicilia MA. (eds) Metadata and Semantics Research. MTSR 2014. Communications in Computer and Information Science, vol 478. Springer, Cham. doi: 10.1007/978-3-319-13674-5_25

The final publication is available at link.springer.com

\title{
Library Data Integration: towards BIBFRAME mapping to EDM
}

\author{
Sofia Zapounidou ${ }^{1}$, Michalis Sfakakis ${ }^{1}$, Christos Papatheodorou ${ }^{1,2}$ \\ ${ }^{1}$ Department of Archives, Library Science and Museology, Ionian University, Corfu, Greece \\ ${ }^{2}$ Digital Curation Unit, IMIS, “Athena” Research Centre, Athens, Greece \\ \{112zapo, sfakakis, papatheodor\}@ionio.gr
}

\begin{abstract}
.
Integration of library data into the Linked Data environment is a key issue in libraries and is approached on the basis of interoperability between library data conceptual models. Achieving interoperability for different representations of the same or related entities between the library and cultural heritage domains shall enhance rich bibliographic data reusability and support the development of new data-driven information services. This paper aims to contribute to the desired interoperability by attempting to map core semantic paths between the BIBFRAME and EDM conceptual models. BIBFRAME is developed by the Library of Congress to support transformation of legacy library data in MARC format into linked data. EDM is the model developed for and used in the Europeana Cultural Heritage aggregation portal.
\end{abstract}

Keywords: Conceptual models, linked data, interoperability, path-oriented mapping, data integration, BIBFRAME, EDM

\section{Introduction}

The advent of the Internet and the World Wide Web emerged powerful tools and possibilities for the development of new, added value information services by museums, libraries and archives. Metadata harvesting and aggregation, as well as linked 
data technologies enforce a shift towards data and data-driven services that enhance the visibility and impact of memory institutions' collections to research, teaching and learning. Aggregation and harvesting presuppose interoperability and therefore there is the apparent need that metadata (i) are expressed by common vocabularies and (ii) their semantics are harmonized with shared and commonly accepted conceptual models.

This paper focuses on libraries and investigates the integration of their data with third party services and their reuse in new contexts. One of the obstacles for integrating library data into the semantic web and publishing them as Linked Data is the existence of different conceptual models and vocabularies. The most well known conceptual models in the library linked data domain are FRBR [1], FRBRoo [2] and BIBFRAME [3].

Newly-developed aggregation services in the cultural heritage $(\mathrm{CH})$ domain collect metadata regarding cultural heritage objects (CHOs) from libraries and other memory institutions aspiring to provide a point of access to $\mathrm{CH}$ information and advanced research supporting services. Two aggregator efforts are in progress in Europe and North America, that of Europeana (http://www.europeana.eu/) and the Digital Public Library of America - DPLA (http://dp.la/). Europeana focuses on European CHOs, while DPLA is oriented to the United States of America. Both projects have developed data models to enable proper harvesting of metadata from a variety of data providers. Europeana provides the Europeana Data model (EDM) [4] and DPLA provides the DPLA Metadata Application Profile [5], which is also based on EDM.

BIBFRAME is the new library data model that the Library of Congress currently develops within the framework of "modeling the MARC 21 format as a Web of Data" $[3,7]$. The Europeana Data Model (EDM) describes the digitized CHOs that the Europeana portal aggregates from European Libraries and other cultural institutions. This paper extends previous work [6] and aims to contribute to interoperability of library data by examining how BIBFRAME core classes and properties could be mapped to EDM according to different paradigms, such as those defined in the library metadata alignment report published in 2012 [8] and the EDM-FRBRoo application profile [18]. For both paradigms contextualized versions of the EDM using the ore:Proxy class [9] are considered, as well as non contextualized versions incorporating the BIBFRAME data directly to the edm:Provided $\mathrm{CHO}$ class.

In the next section BIBFRAME and EDM conceptual models are briefly presented, while section 3 describes the methodology followed for the proposed mapping and provides a test case of seven library records. Section 4 presents proposed mappings between the two models following different scenarios and Section 5 discusses and concludes the derived results.

\section{Background}

Libraries typically describe their holdings using MARC format [7]. MARC records provide information regarding the physical copies held at a library to enable searching and locating on the shelves. On the occasion of the physical copy more information is 
added to each record, such as the intellectual content of the physical item, bibliographic details regarding the publication process, custodial history, relationships between and among other bibliographic entities, subjects, etc. [2, 8]. The flat record structure in MARC presents many insufficiencies and has been criticized by experts and library-related international organizations [10-14]. Moreover it does not facilitate meaningful representation and interchange of bibliographic data in the semantic web environment.

Library of Congress announced in 2011 [15] its decision to "experiment with Semantic Web and linked data technologies to see what benefits to the bibliographic framework they offer our community and how our current models need to be adjusted to take fuller advantage of these benefits." BIBFRAME is the new model that the Library of Congress develops within the context of the bibliographic framework initiative. Its main classes are: Creative Work, Instance, Authority and Annotation [3]. The class Creative Work (or simply Work) reflects the "conceptual essence of the cataloguing item" [3]. The class Instance reflects "an individual, material embodiment of the Work". The class Authority is used to identify People, Places, and Organizations involved in the creation or publication of a Work. For the expression of topics, BIBFRAME Authority simply works as a linking mechanism to LC Subject Headings published as linked data ${ }^{1}$. The class Annotation expresses comments made about a BIBFRAME Work, Instance, or Authority. Examples of BIBFRAME annotations are: library holdings, cover arts, sample texts, reviews, etc. (see Figure 1).

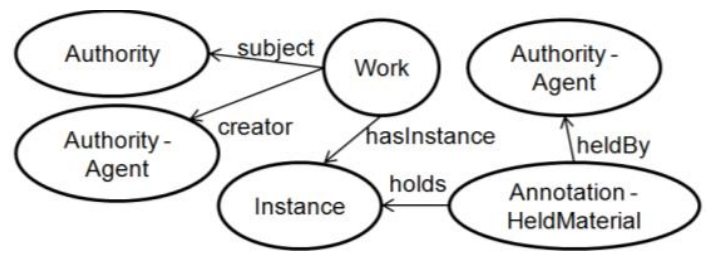

Fig. 1. BIBFRAME model with Annotation for holding

Europeana aggregates metadata about and enables access to digital cultural heritage resources provided by European memory institutions. Descriptions over Europeana are made with Europeana Semantic Elements [16], a basic data model that uses Dublin Core's 15 elements and other 12 additional elements. EDM has been developed for the better semantic expression of the cultural heritage descriptions that Europeana data providers contribute. No community-driven standard was used as a basis for its development and the Semantic Web framework was taken into account [4].

EDM's scope is wider than BIBFRAME's; thus different semantics and abstraction layers are used. For each provider, EDM distinguishes between real provided CHOs and their digital representations, and between provided CHOs and their descriptions. Europeana collects only descriptions for objects having at least one web representation [4]. EDM provides three core classes, namely edm:ProvidedCHO (for provided Cultural Heritage Object), edm:WebResource (for the edm:ProvidedCHO digital rep-

${ }^{1}$ http://id.loc.gov/ 
resentations) and ore:Aggregation (for the aggregation of the activities made by the provider of the edm:ProvidedCHO).

The alignment of EDM to library metadata is a work in progress. The library metadata alignment report published in 2012 [8] focuses on specific library materials (monographs, multi-volume works and serials), does not adopt current bibliographic records' flat structure and adheres to linked data principles. A key point for the development of the report was the separation of the item in hand (e.g. the book) from its edition which represents the entirety of all identical copies of the item. Despite the fact that the need for compliance with FRBR was recognized by the report, the concept 'edition' was introduced as the union of FRBR Work, Expression and Manifestation entities and thus the desired compliance was postponed.

According to the report the 'edition' level information of the resource is represented by the edm:ProvidedCHO class, while the digital representation of the real world object is represented by the edm:WebResource class. The ore:Aggregation class links the description of the provided resource with its digital representations.

EDM in order to contextualize harvested descriptions of the same $\mathrm{CHO}$ provided by different institutions utilizes the ore:Proxy class [17]. The added value of using proxies is that there will be only one edm:ProvidedCHO class instance for each European Heritage object along with multiple instances of the ore:Proxy class, provided by different providers. Hence a provider's description (metadata) is assigned as properties at the ore:Proxy class (see Figure 2). It is worth mentioning that the ore:Proxy class was not considered by [8].

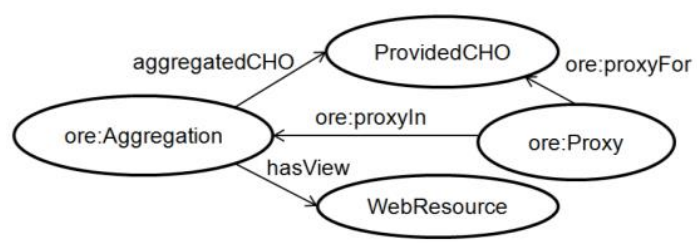

Fig. 2. Europeana data model with use of ore:Proxy

The report on alignment of library data to EDM [8] concluded that it should be considered as a milestone and that it needed to be reviewed to "integrate the FRBR entities in EDM using FRBRoo terms". This provision prompted the launch of the EDM-FRBRoo application profile Task Force in July 2012 which completed its activities in April 2013 announcing an application profile [18]. The Task Force in order to translate classes of the FRBR model to EDM, without the introduction of new specialized classes in EDM considered FRBR classes as skos: Concept class instances (Figure 3) and then, related edm:InformationResource classes with the FRBR vocabulary using the edm:hasType property. In Figure 3 an example of the translation of FRBR Group 1 Work and Expression entities as concepts in EDM is shown. 


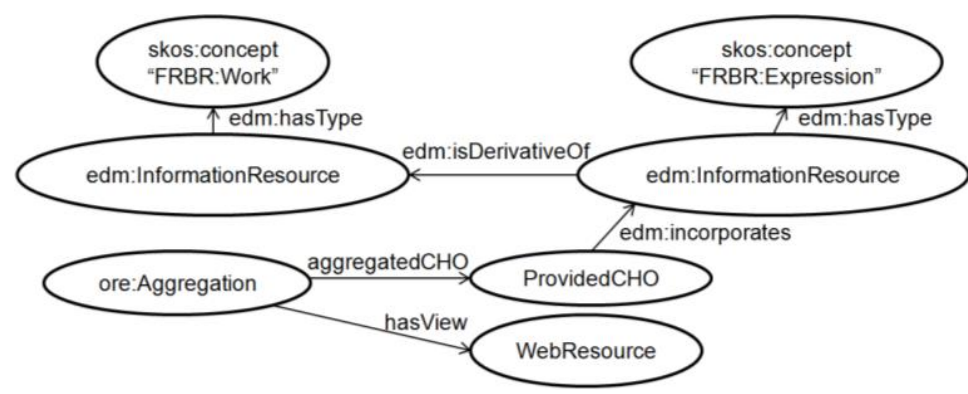

Fig. 3. FRBR Group 1 Work and Expression concepts expressed with EDM classes in the EDM-FRBRoo application profile [18]

Transforming instances from BIBFRAME into EDM, with respect to the above alternative frameworks, will examine key issues in interoperability between the two models, as well as will suggest semantic alignments between the intentions of the two models' communities.

\section{Integration scenarios - requirements}

The methodology adopted in this work is a combination of the ones used in the Europeana Libraries project [8] for the alignment of library metadata with the Europeana Data Model and the EDM - FRBRoo Application Profile Task Force [18]:

1. Selection of specific type(s) of library material

2. Definition of requirements for a BIBFRAME - EDM profile

3. Selection of a real test case and bibliographic records

4. Representation(s) of the test case in BIBFRAME

5. Attempt for a BIBFRAME - EDM mapping following a path-oriented approach

6. Mapping of BIBFRAME representations into EDM using different modeling patterns described in the next paragraph.

The most common library material type is monographs, and therefore this paper focuses on monographs and multivolume works. Different types of monographs include -but are not limited to- simple monographs, multipart monographs, derivations of a monograph such as translations, adaptations, etc, reproductions and aggregates. These categories of monographs are estimated in millions of records in WorldCat according to two studies performed in 2002 [22] and in 2011 [23]. The requirements for our BIBFRAME - EDM mappings are defined as follows:

- BIBFRAME - EDM mappings will be performed using different EDM modeling paradigms; namely the definitions of the Europeana Data Model for Libraries report [8], use of ore:Proxy class as described in [17] (see Figure 2), use of edm:InformationResource class as used in [18] (see Figure 3) and concurrent use of the edm:InformationResource and ore:Proxy classes (see Figures 2 and 3). 
- BIBFRAME is a linked data model. Therefore the BIBFRAME-EDM profile shall use Resource Description Framework syntax and shall support the use of URIs.

- The BIBFRAME-EDM mappings shall be flexible enough to enable meaningful representations for other types of library material.

Cervantes' "Don Quixote" has been selected as our test case, because it may exemplify different types of monographs, as referred above, enabling complex representations in BIBFRAME and scrutiny of possible BIBFRAME-EDM mappings. Therefore use of the "Don Quixote" case shall enable identification and study of representation issues in BIBFRAME for whole monograph categories, as well as their mapping into EDM.

"Don Quixote" consists of two separate works: the first one entitled "El ingenioso hidalgo don Quixote de la Mancha" was published in 1605 and the second one entitled "Segunda parte del ingenioso cauallero don Quixote de la Mancha" was published in 1615. These two parts have been published, translated and reproduced afterwards as independent volumes, as well as in a single volume. Moreover, there are many adaptations, as well as other works based on variations of the original work. Our test case consists of seven bibliographic records from the National Library of Spain and the Library of Congress that describe (i) the first editions of the two parts (denoted as 'First part' and 'Second part' respectively in Figure 4), (ii) the first edition that incorporated both parts (denoted as 'Two parts'), (iii) a French translation of both parts (denoted as 'French translation'), (iv) an English translation that was based on the former French one (denoted as 'English translation'), (v) an annotated edition of both parts by the Cervantes Institute (denoted as 'Annotated edition') and (vi) a CD-ROM (denoted as 'CD-ROM') that compiled the annotated edition's text with a linguistic database developed on this content. The linguistic database is also represented in BIBFRAME and EDM representations. It must be noted that some of the mentioned records are the same to the ones used by the EDM-FRBRoo Application profile task force [18]. Yet, we selected a few additional records from the National Library of Spain and the Library of Congress to study specific representation cases, such as reproduction and aggregates. 


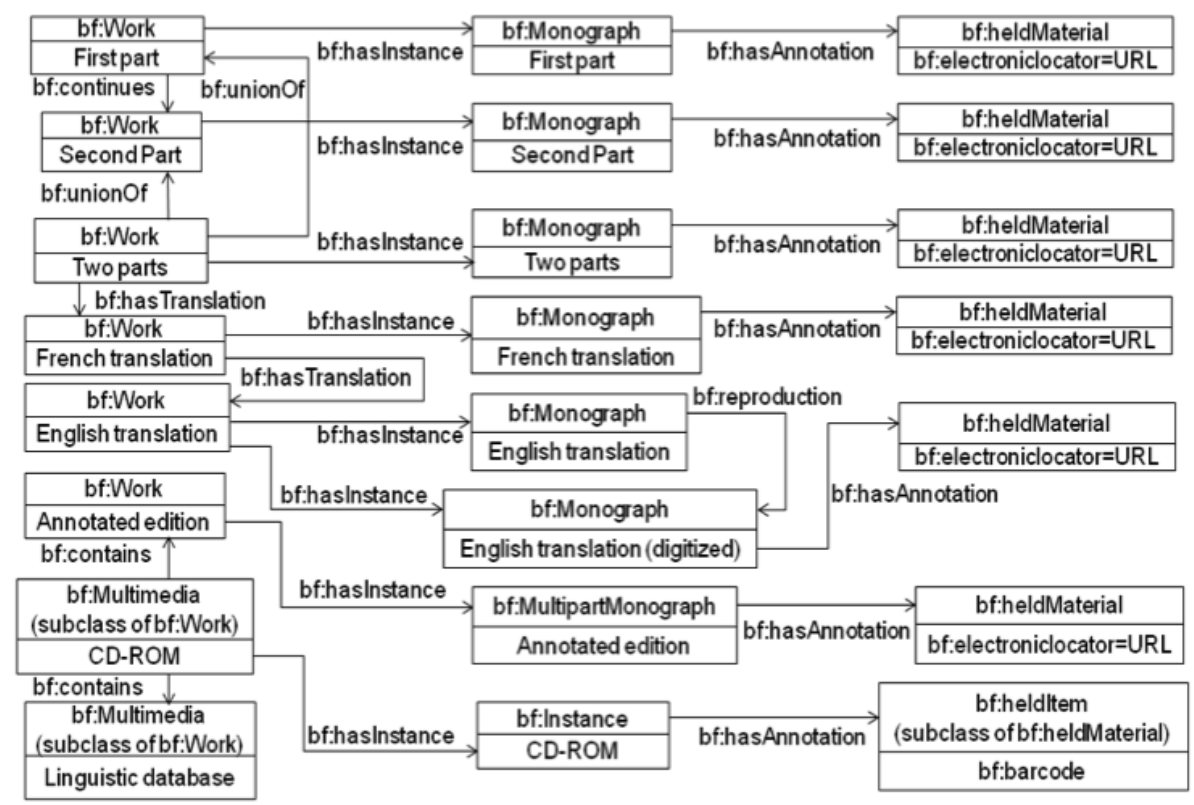

Fig. 4. BIBFRAME representation of the "Don Quixote" test case

In BIBFRAME every record from our sample corresponds to a $b f$ :Work class linked with the respective embodiment Instance or subclass of Instance (e.g. bf:Monograph). Thus, eight individual works are generated to represent the intellectual content of the two independent volumes, the single volume publication for both parts, its translations in English and in French, the Cervantes Institute's annotated edition, the linguistic database derived from the Cervantes' annotated edition and the CD-ROM containing (bf:contains) both the Cervantes' edition with the linguistic database (Figure 4). The relationships between the parts are implemented using the partOf relation of the Work class. All instances, except the CD-ROM, are digitized (bf:heldMaterial class with a bf:electronicLocator property) and may be openly accessed online.

Mapping between distinct models that serve different purposes is not a straightforward issue. Therefore we decided to elucidate the semantics of each model and of their mappings following a path-oriented approach [19-21]. Paths are defined as sequences of "domain class - property - range class" statements and enhance comprehensibility of each model's semantics. The paths of the source model (in our case BIBFRAME) are mapped to semantically equivalent paths of the target model (in our case EDM).

Mappings between BIBFRAME and EDM follow different paradigms that may serve distinct library organizations', collections' or end-users' requirements. At first the Don Quixote's test case BIBFRAME representation is mapped to the EDM applying the definitions expressed in the EDM library data alignment report [8]. Then the contextualization of CHOs' ingested descriptions is examined with the use of the 
ore:Proxy class as defined in [17]. Examining the transformation of BIBFRAME to EDM with use of the edm:InformationResource class follows. This alternative mapping is based upon the pattern used in the EDM-FRBRoo application profile [18], highlighted in section 2. The final scenario examined is concurrent use of edm:InformationResource and ore:Proxy classes (Figures 2 and 3).

\section{Mapping BIBFRAME to EDM}

Europeana aggregates descriptions of CHOs in digital form only. In BIBFRAME the existence of a library object that is in digital form and therefore may be aggregated by Europeana is expressed by the following path "Work -hasInstance - Instance - hasAnnotation - heldMaterial - electronicLocator - URI". In this paper's framework this path is considered the basic BIBFRAME path from which four different mappings to EDM are attempted. It is worth mentioning that in case where EDM is used as the conceptual model for an information integration system and not for aggregating descriptions in the Europeana portal, the basic BIBFRAME path might be slightly different due to the inclusion of non digital materials. This paradigm is out of the scope of this work and is suggested as a further extension to these mappings.

\subsection{Mapping according to the EDM library data alignment report}

The EDM library data alignment report was published in 2012 with the aim to "describe how library metadata can be aligned with the EDM" [8]. This report referenced the FRBR WEMI [1] entities (Work, Expression, Manifestation, Item) but did not achieve a one-to-one mapping to EDM classes. Yet it introduced the concept of "edition" to include "all information concerning the Manifestation, Expression and Work entities" and defined that the edm:ProvidedCHO class is at this "edition level". The edm:WebResource class is defined in this report's framework as the "digital representation of an item".

In BIBFRAME information regarding the intellectual content and its expression (Work and Expression entities in FRBR) is at the Creative Work class level. Information regarding the publishing product/object is at the FRBR Manifestation level and is expressed in BIBFRAME through the Instance class. Holdings (Items in FRBR) are stated through the following path "Instance - hasAnnotation - heldMaterial - ...". Therefore the basic BIBFRAME path may be mapped to EDM as Figure 5 demonstrates.

The path "Work -hasInstance - Instance" is mapped to a single ProvidedCHO instance, and selected properties from the $b f$ :Work and bf:Instance could be mapped to similar ProvidedCHO properties. Existence of the basic BIBFRAME path justifies an instantiation of the edm:WebResource class, with id the URI from the BIBFRAME path. For more details describing this paradigm see our previous work in [6]. 


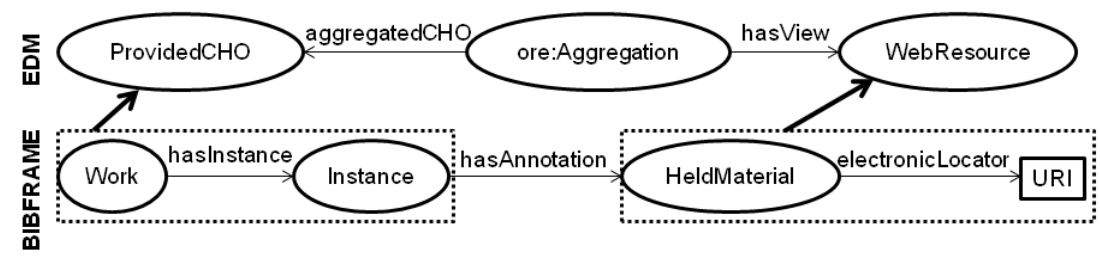

Fig. 5. Mapping of the basic BIBFRAME path to core EDM classes, according to the library metadata alignment report [8]

\subsection{Use of ore:Proxy class}

As already mentioned, EDM uses the ore:Proxy class to contextualize the CHOs' harvested descriptions. Thus descriptions (metadata) submitted by different organizations will be preserved with use of the ore:Proxy class. In this case the BIBFRAME "Work -hasInstance - Instance" path is mapped to a single ore:Proxy class instance. It must be noted that any relationships between BIBFRAME Work class instances will be most likely mapped to ore:Proxy class instances and not to edm:ProvidedCHO class instances. Similarly to the previous mapping approach, the BIBFRAME path describing a born-digital or digitized library object justifies an instantiation of the edm:webResource class, with id the URI from the BIBFRAME path (Figure 6).

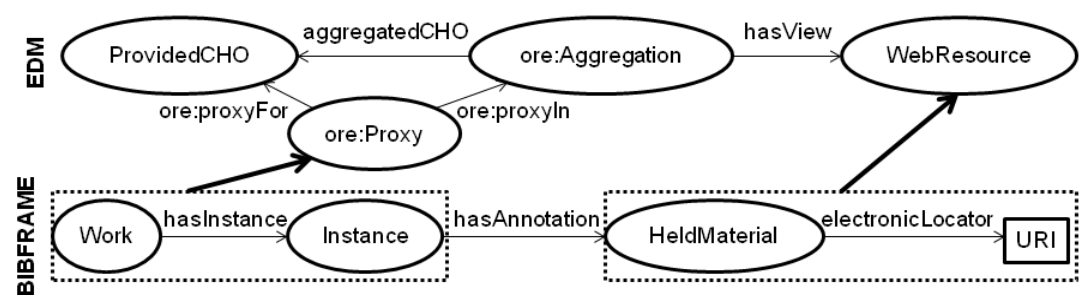

Fig. 6. Mapping of the basic BIBFRAME path to core EDM classes using the ore:Proxy class

\subsection{Use of edm:InformationResource class}

EDM was developed as community agnostic model. It serves as the model to aggregate descriptions of digital representations of CHOs mainly for the Europeana portal, while BIBFRAME serves as a model for describing library materials according to the library community objectives. EDM-FRBRoo profile [18] was developed to fulfill the need expressed in [8] for integration of FRBR semantics in EDM using FRBRoo [2] terms.

In this EDM - FRBRoo Application Profile more FRBR semantics may be expressed for an instance of the edm:ProvidedCHO class, as described in section 2. FRBR Works -and respectively Expressions- are represented by the path "edm:InformationResource - edm:hasType - frbr:Work". As depicted in figure 7, we propose BIBFRAME Work to be mapped to the edm:InformationResource class typed as bf:Work, where bf:Work is a skos:Concept instance related to 
edm:InformationResource by the edm:hasType property. Therefore a BIBFRAME Work is mapped to the "edm:InformationResource - edm:hasType - bf:Work" EDM path. Instance reflects "an individual, material embodiment of the Work" is mapped to the edm:ProvidedCHO. It must be noted that since in this mapping there exists a higher level of abstraction (edm:InformationResource class) mapped to the $b f$ :Work class all relationships between Works in BIBFRAME are going to be expressed between the respective edm:InformationResource instances.

The transformation of the BIBFRAME representation (see Figure 4) of the "Don Quixote" test case to EDM using typed edm:InformationResource class is presented in Figure 8 proving that successful mapping may be achieved for core BIBFRAME classes and properties. This scenario may support translation of different BIBFRAME paths besides the basic one defined earlier. The CD-ROM object along with the linguistic database are not available online and are represented at a more abstract level, as edm:InformationResource class instances.

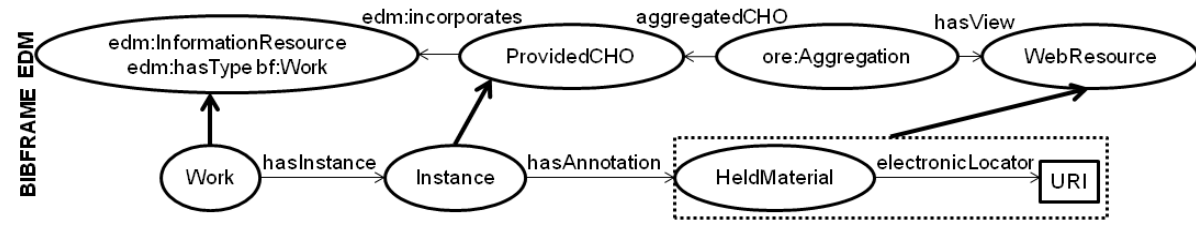

Fig. 7. Mapping of the basic BIBFRAME path to EDM using typed edm:InformationRecource class

\subsection{Use of edm:InformationResource and ore:Proxy classes}

In order to maintain and preserve information regarding the conceptual content included in provided CHOs along with different providers' views the scenario of using both edm:InformationResource and ore:Proxy classes was identified. In this case a BIBFRAME Work class instance is mapped to the "edm:InformationResource edm:hasType - skos:Concept - bf:Work" EDM path instance, while a BIBFRAME Instance class instance is mapped to an ore:Proxy class instance (Figure 9). 


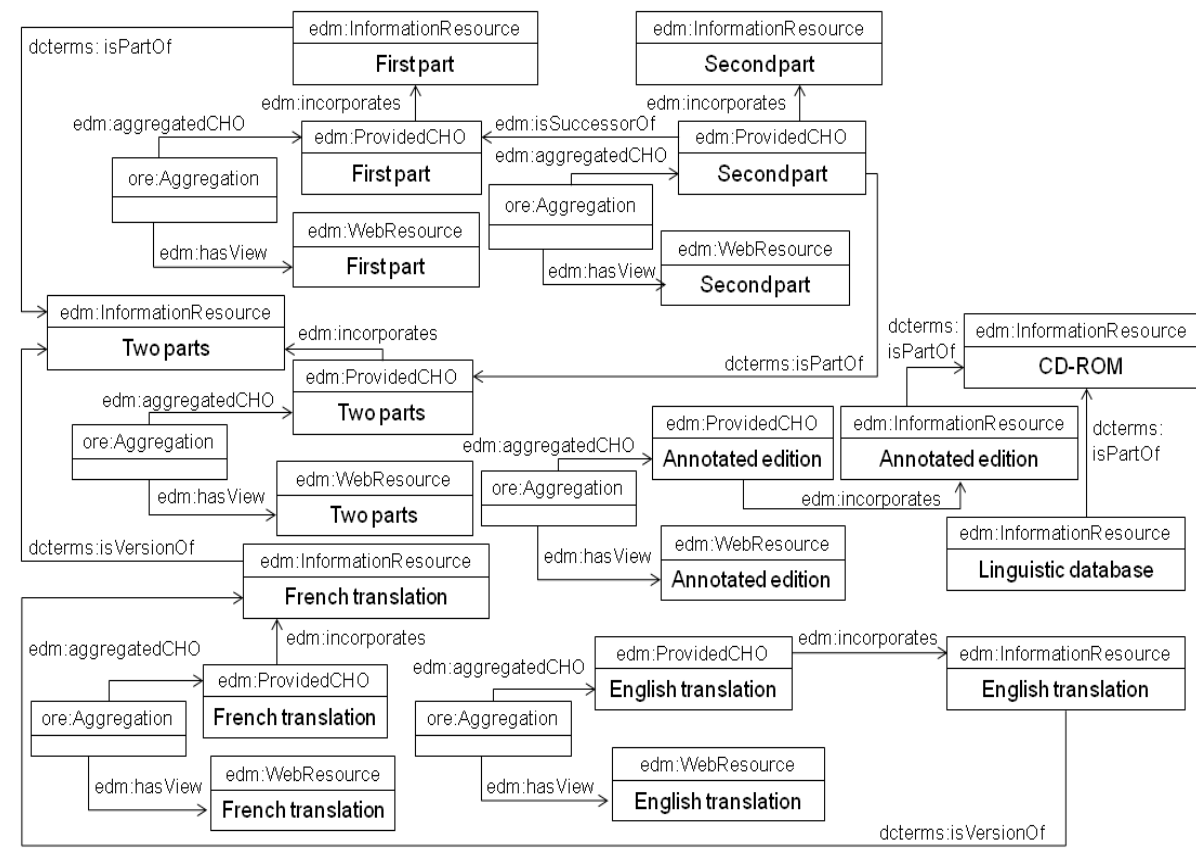

Fig. 8. "Don Quixote" test case in EDM using typed edm:InformationRecource class

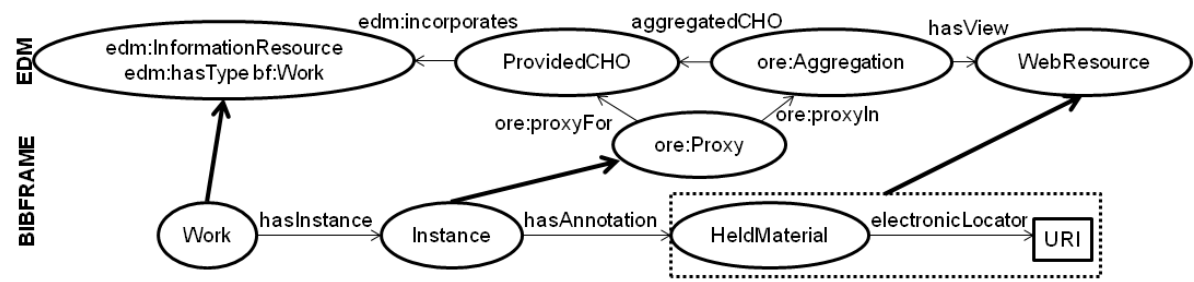

Fig. 9. Mapping of the basic BIBFRAME path to EDM using the edm:InformationRecource and ore:Proxy classes

\section{Discussion and Conclusions}

Libraries are expected to engage in interoperability-related activities to enable integration of their bibliographic data into the Semantic Web and their re-use by thirdparty services. This prospect has motivated our work to attempt translating BIBFRAME conceptualization in the Europeana framework using EDM classes and properties. The mapping of BIBFRAME core classes and properties to EDM has been developed taking into consideration different scenarios expressed as alternative EDM representations; use of the library data alignment report that introduced the "edition" concept [8], use of ore:Proxy class that preserves context of CHOs' ingested descriptions, use of edm:InformationResource class according to the EDM-FRBRoo application profile and concurrent use of edm:InformationResource and ore:Proxy classes to 
better express BIBFRAME semantics and preserve providers' view (descriptive metadata) about the holdings-CHOs they provide to Europeana.

In our mapping a path approach was adopted to avoid possible semantic misinterpretations. Use of source and target paths furnished explicit semantic expressions and mappings between the source BIBFRAME and the target EDM data models, as defined in models' current specifications. Mapping was attempted between a basic BIBFRAME path and the EDM alternative representations. A basic BIBFRAME path was defined in order to satisfy Europeana's focus on born-digital or digitized CHOs available online. BIBFRAME "Work -hasInstance - Instance - hasAnnotation heldMaterial - electronicLocator - URI" paths of the "Don Quixote" test case were successfully mapped to all possible EDM representation alternatives, proving that various modeling requirements may be satisfied. In case where EDM is used as the conceptual model for an information integration system and not only for aggregating descriptions in the Europeana portal, the above mappings of the BIBFRAME paths require further investigation in order to incorporate descriptions of non digital materials utilizing among others the edm:PhysicalThing class.

This is a preliminary work regarding mapping of the core BIBFRAME classes and properties to EDM. More investigations are needed to include more paths and to provide a full application profile. Both models are evolving since BIBFRAME is under development and EDM is regularly updated. Therefore a future application profile should take into account possible changes in each model's semantics. Besides the fact that "Don Quixote" may be considered as a representative example for whole categories of monographs, additional tests need to be performed with a larger set of bibliographic records that will also include more types of aggregates, as well as other types of library materials, such as serials, cartographic material, ephemera, collections, graphic material, etc.

\section{$6 \quad$ References}

1. IFLA Study Group on the Functional Requirements for Bibliographic Records: Functional Requirements for Bibliographic Records. Final Report. IFLA, The Hague (2009), http://www.ifla.org/files/assets/cataloguing/frbr/frbr_2008.pdf

2. Bekiari, C., Doerr, M., Bœuf, P. Le, Riva, P.: FRBR object-oriented definition and mapping from FRBRER, FRAD and FRSAD (v.2.0). International Working Group on FRBR and CIDOC CRM Harmonisation, Paris (2013), http://www.cidoccrm.org/frbr_drafts.html

3. Miller, E., Ogbuji, U., Mueller, V., MacDougall, K.: Bibliographic Framework as a Web of Data: Linked Data Model and Supporting Services. Report, Library of Congress, Washington, DC (2012), http://www.loc.gov/bibframe/pdf/marcld-report-11-21-2012.pdf

4. Isaac, A.: Europeana Data Model Primer. Europeana Project, The Hague (2013), http://pro.europeana.eu/edm-documentation

5. Digital Public Library of America: Metadata Application Profile, Version 3. Report, Digital Public Library of America, Boston, MA (2013), http://dp.la/info/developers/map/

6. Zapounidou, S., Sfakakis, M., Papatheodorou, C.: Integrating library and cultural heritage data models: the BIBFRAME - EDM case. To be appeared in: Panhellenic Conference on Informatics Proceedings, Athens (2014), http://dx.doi.org/10.1145/2645791.2645805 
7. Library of Congress: MARC standards, http://www.loc.gov/marc/

8. Angjeli, A., Bayerische, M., Chambers, S., Charles, V., Clayphan, R., Deliot, C., Eriksson, J., Freire, N., Huber, A., Jahnke, A., Pedrosa, G., Phillips, V., Pollecutt, N., Robson, G., Seidler, W., Rühle, S.: D5.1 Report on the alignment of library metadata with the European Data Model (EDM) Version 2.0. Report, Europeana Project (2012), http://www.europeana-libraries.eu/web/guest/outcomes

9. Lagoze, C., Sompel, H. Van De, Johnston, P., Foundation, E., Nelson, M., Sanderson, R., Warner, S.: Open Archives Initiative Object Reuse and Exchange ORE Specification Abstract Data Model. (2008), http://www.openarchives.org/ore/1.0/datamodel

10. Heaney, M.: Object-oriented cataloging. Information Technology and Libraries. 14, 135153 (1995)

11. Weihs, J. (ed.) International Conference on the Principles and Future Development of AACR Toronto, Canada, October 23-25, 1997. Canadian Library Association; Library Association Publishing; American Library Association, Ottawa; London; Chicago (1998)

12. IFLA Study Group on the Functional Requirements for Bibliographic Records: Functional Requirements for Bibliographic Records Final Report. Saur, München (1998)

13. Tennant, R.: A Bibliographic Metadata Infrastructure for the 21st Century. Library Hi Tech. 22, 175-181 (2004)

14. Calhoun, K.: The changing nature of the catalog and its integration with other discovery tools. Report, Library of Congress (2006)

15. Deanna B. Marcum: Transforming our Bibliographic Framework: A Statement from the Library of Congress (May 13, 2011), http://www.loc.gov/bibframe/news/index.html

16. Europeana Semantic Elements Specification and Guidelines. Report, Europeana Project (2013), http://pro.europeana.eu/ese-documentation/

17. Definition of the Europeana Data Model v5.2.5. Report, Europeana Project (2014), http://pro.europeana.eu/edm-documentation

18. Doerr, M., Gradmann, S., Leboeuf, P., Aalberg, T., Bailly, R., Olensky, M.: Final Report on EDM-FRBRoo Application Profile Task Force. Report, Europeana Project (2013), http://pro.europeana.eu/web/network/europeana-tech

19. Kondylakis, H., Doerr, M., Plexousakis, D.: Mapping Language for Information Integration. Technical report 385, ICS - FORTH, Heraklion, Crete, Greece (2006), http://www.ics.forth.gr/isl/index_main.php?l=e\&pbl=ISL\&lab=ISL\&author=Doerr

20. Stasinopoulou, T., Bountouri, L., Kakali, C., Lourdi, I., Papatheodorou, C., Doerr, M., Gergatsoulis, M.: Ontology-based metadata integration in the cultural heritage domain.In: Goh, D.H.L., Cao, H.T., Sølvberg, I., Rasmussen, E. (Eds.): ICADL 2007. LNCS 4822, pp. 165-175. Springer, Heidelberg (2007)

21. Lourdi, I., Papatheodorou, C., Doerr, M.: Semantic integration of collection description. D-Lib Magazine. 15 (2009)

22. Bennett, R., Lavoie, B.F., O’Neill, E.T.: The Concept of a Work in WorldCat: An Application of FRBR. Library Collections, Acquisitions and Technical Services 27, 45-59 (2003)

23. Žumer, M., O’Neill, E.T.: Modeling Aggregates in FRBR. Cataloging \& Classification Quarterly . 50, 456-472 (2012) 